\title{
Human Adipose Tissue-Derived Mesenchymal Stem Cells in Parkinson's Disease: Inhibition of T Helper 17 Cell Differentiation and Regulation of Immune Balance Towards a Regulatory T Cell Phenotype
}

This article was published in the following Dove Press journal: Clinical Interventions in Aging

\author{
Yong $\mathrm{Bi}^{1-3}$ \\ Xiaobin $\operatorname{Lin}^{4}$ \\ Huazheng Liang $\mathbb{D}^{2}$ \\ Dehao Yang (D) ${ }^{4}$ \\ Xiaowei Zhang ${ }^{4}$ \\ Jianming $\mathrm{Ke}^{4}$ \\ Jingjing Xiao $\mathbb{D}^{2}$ \\ Zhilin Chen $\mathbb{D}^{2}$ \\ Weian Chen ${ }^{4}$ \\ Xu Zhang ${ }^{4}$ \\ Shaoshi Wang ${ }^{2}$ \\ Chun-Feng Liu $\mathbb{D}^{1,3}$ \\ 'Department of Neurology and Suzhou \\ Clinical Research Center of Neurological \\ Disease, The Second Affiliated Hospital \\ of Soochow University, Suzhou, People's \\ Republic of China; ${ }^{2}$ Department of \\ Neurology, Translational Research \\ Institute of Brain and Brain-Like \\ Intelligence, Shanghai Fourth People's \\ Hospital Affiliated to Tongji University \\ School of Medicine, Shanghai, People's \\ Republic of China; ${ }^{3}$ Institute of \\ Neuroscience, Soochow University, \\ Suzhou, People's Republic of China; \\ ${ }^{4}$ Department of Neurology, The First \\ Affiliated Hospital of Wenzhou Medical \\ University, Wenzhou, People's Republic \\ of China
}

Background: Parkinson's disease (PD) is a neurodegenerative disorder displaying a typical neuroinflammation pathology that may result from an imbalance between regulatory $\mathrm{T}$ cells (Treg) and T helper 17 (Th17) cells. Human adipose tissue-derived mesenchymal stem cells (AdMSCs) exert immunomodulatory effects by inhibiting effector $\mathrm{T}$ cell responses and have been used to treat diverse immune disorders. We aimed to investigate the modulating effect of human Ad-MSCs on peripheral blood mononuclear cells (PBMCs) of patients with PD, focusing on differentiation into Th17 and Treg cells.

Methods: We isolated human peripheral blood $\mathrm{CD}^{+} \mathrm{T}$ cells and co-cultured them with Ad-MSCs at a ratio of 4:1 under either Th17 or Treg cell polarizing conditions for 4 days to detect the proportions of IL-17-producing $\mathrm{CD} 4{ }^{+} \mathrm{T}(\mathrm{Th} 17)$ and $\mathrm{CD} 4{ }^{+} \mathrm{CD} 25^{+}$Foxp $3^{+}$regulatory $\mathrm{T}$ (Treg) cells by flow cytometry. We also determined the mRNA expression levels of the retinoid-related orphan nuclear receptor $(\mathrm{ROR} \gamma \mathrm{t})$ transcription factor and those of interleukin-6 receptor (IL-6R), interleukin-23 receptor (IL-23R), leukemia inhibitory factor (LIF), and LIF receptor (LIFR) by quantitative reverse transcription PCR. We detected levels of cytokines in the supernatant (including LIF, IL-6, IL-23, IL-10, and TGF- $\beta$ ) using ELISA.

Results: Our results showed that Ad-MSCs specifically inhibited the differentiation of PBMCs of patients with PD into IL-17-producing $\mathrm{CD}^{+} \mathrm{T}$ cells by decreasing expressions of IL-6R, IL-23R, and ROR $\gamma \mathrm{t}$ (the key transcription factor for Th17 cells). Moreover, AdMSCs induced a functional $\mathrm{CD} 4{ }^{+} \mathrm{CD} 25^{+} \mathrm{Foxp} 3^{+} \mathrm{T}$ regulatory cell phenotype as evidenced by the secretion of IL-10. The levels of IL-6, IL-23, and TGF- $\beta$ remained constant after coculture under either the Th17 or the Treg cell polarizing condition. In addition, levels of LIF protein and its receptor mRNA were significantly increased under both polarizing conditions. Conclusion: The present in vitro study found that Ad-MSCs from healthy participants were able to correct the imbalance between Th17 and Treg found in PBMCs of PD patients, which were correlated with an increase in LIF secretion and a decrease in expression of IL-6R, IL23R, and ROR $\gamma$ t. These findings should be confirmed by in vivo experiments.

Keywords: Parkinson's disease, adipose-derived mesenchymal stem cells, CD4 ${ }^{+} \mathrm{T}$ cell, $\mathrm{T}$ helper 17 cell, T regulatory cell, peripheral blood mononuclear cells, leukemia inhibitory factor

\section{Introduction}

Parkinson's disease (PD) is a chronic neurodegenerative disorder characterized by progressive loss of dopaminergic neurons due to Lewy pathology and the presence of an active inflammatory response leading to classical motor and non-motor
Correspondence: Chun-Feng Liu Email liuchunfeng@suda.edu.cn 
symptoms. ${ }^{1,2}$ Some studies have indicated that $\mathrm{T}$ helper cells may contribute to the cascade of neuroinflammation by breaking the balance between peripheral T helper 17 (Thl7) and regulatory $\mathrm{T}$ (Treg) cells. ${ }^{3-9}$ IL-17-producing Th17 cells contribute to cell death of nigrostriatal dopaminergic neurons, whereas Treg cells attenuate these effects. ${ }^{10,11}$ As a result, an imbalance between Th17 and Treg cells leads to neurodegeneration in the substantia nigra.

Researchers have found increased numbers of $\mathrm{T}$ lymphocytes in postmortem brain tissues of patients with PD and increased numbers of Th17 cells in their peripheral blood. ${ }^{3-6,8}$ Mice intoxicated with 1-methyl4-phenyl-1,2,3,6-tetrahydropyridine (MPTP) have shown similar characteristics. ${ }^{11-14}$ Increased numbers of Th17 cells in the blood of patients with PD were correlated with the death of human-induced pluripotent stem cell (hiPSC)-derived midbrain neurons. ${ }^{10}$ In contrast, Treg cells were found to attenuate inflammatory responses, activate microglial cells, and to protect dopaminergic neurons from MPTP intoxication. ${ }^{12,15,16}$ Dysfunctional or few Treg cells in the blood of patients with PD cause imbalanced immune responses. ${ }^{5,17-20} \mathrm{An}$ imbalance between Th17 and Treg cells found in adaptive immunity may be part of the pathogenesis of PD.

Th17 cells express the transcription factor ROR $\gamma \mathrm{t}$ and secrete IL-17. ${ }^{21}$ Their effects are counteracted by Foxp $3^{+}$ Treg cells that express the transcription factor Foxp3 and secrete IL- $10 .{ }^{22}$ In the presence of IL- 6 and TGF- $\beta$, $\mathrm{CD} 4{ }^{+} \mathrm{T}$ cells increase their expression of IL-23R and ROR $\gamma$ t, which in turn suppress the expression of Foxp $3^{22,23}$ and the production of IL-10. ${ }^{23}$ The progression of the inflammatory response may be associated with an increased number of Th17 cells and a decreased number of Treg cells. ${ }^{22-24}$ Despite extensive efforts in investigating the PD pathogenesis, no treatments can stop the progression of this disease. Thus, identifying factors that inhibit Th17 cell differentiation and induce Treg cell differentiation to achieve an immune balance and suppress the inflammatory response may be a promising therapeutic option for patients with PD.

Adipose tissue-derived mesenchymal stem cells (AdMSCs) are promising candidates due to the ease of access to autologous adipose tissues, their abundance, proliferation, differentiation potential, and their immunoregulatory capacity to suppress inflammatory responses. ${ }^{24,25}$ Moreover, Ad-MSCs have been used to regulate immune responses in various conditions, ${ }^{26-29}$ and their effectivity may be explained by their capability of inducting Treg and suppressing Th17 cell differentiation. ${ }^{9,12,18,27,29,30}$ This mechanism might be beneficial for the management of patients with PD.

Our studies on multiple sclerosis (MS) and its mouse model have shown that Ad-MSCs alleviate inflammatory responses through the above-mentioned mechanism by specifically secreting LIF. ${ }^{31,32}$ However, whether Ad-MSCs can induce $\mathrm{CD}^{+}{ }^{+} \mathrm{T}$ to differentiate into Treg cells and can regulate immune responses in patients with PD remains unclear. Considering that Th17/Treg imbalance is associated with disease progression in patients with PD and that Ad-MSCs may provide a promising therapeutic approach for PD (through their regulation of different immune cells), confirming whether Ad-MSCs upregulate Treg cells and downregulate Th17 cells of PBMCs of patients with PD is important. In the present study, we aimed to investigate the effects of AdMSCs on PBMCs of patients with PD, focusing on the differentiation of $\mathrm{CD} 4^{+} \mathrm{T}$ cells and the expression of relevant transcription factors and cytokines.

\section{Materials and Methods Isolation, Culture, and Identification of Human MSCs}

We obtained adipose tissue samples from 7 women (ranging from 22-35 years) undergoing plastic surgery at the Department of Plastic Surgery of the First Affiliated Hospital of Wenzhou Medical University. We identified potential tissue donors after excluding those with infectious diseases, autoimmune disorders, or other severe medical conditions. The Human Research Ethics Committee of our hospital approved the procedures for this study (WYYY2014-001) and we obtained written informed consent from all the donors. This study was conducted in accordance with the Declaration of Helsinki. Briefly, the adipose tissue specimens were sliced for culture in low-glucose Dulbecco's Modified Eagle Medium (LG-DMEM; HyClone, Logan, UT, USA) with $10 \%$ fetal bovine serum (FBS; Gibco, Rockville, MD, USA) and 1\% penicillin/streptomycin (Solarbio, Beijing, China) at $37^{\circ} \mathrm{C}$ with $5 \% \mathrm{CO}_{2}$ and $95 \%$ air for $72 \mathrm{~h}$. After 5-7 days, we identified migrated spindle-shaped Ad-MSCs on the bottom of the culture flasks. We passaged the cells once $80 \%$ confluence was reached. The presence of cell surface markers was assessed using flow cytometry to confirm the identity of the cells.

\section{Isolation and Co-Culture of CD4 ${ }^{+} \mathrm{T}$ Cells with Ad-MSCs}

We isolated $\mathrm{CD}^{+} \mathrm{T}$ cells (purity $95 \%$ ) from freshly collected peripheral blood from six patients with PD in the 
Department of Neurology at the First Affiliated Hospital of Wenzhou Medical University, who met diagnostic PD criteria (UK Brain Bank, London, UK) ${ }^{2}$ using a $\mathrm{CD}^{+}{ }^{+}$T cell isolation kit (Miltenyi Biotechnology, Bergisch, Gladbach, Germany, Cat No.130-094-131). We added anti-CD4-PEcy5.5 to the final cell suspension and selected an appropriate column and sorter for flow cytometry. We stimulated $\mathrm{CD} 4{ }^{+} \mathrm{T}$ cells with a $\mathrm{CD} 3 / \mathrm{CD} 28$ antibody to proliferate and to differentiate into Th17 cells using interleukin- $1 \beta$ (IL-1 $\beta$ ), interleukin-6 (IL-6), transforming growth factor- $\beta$ (TGF- $\beta$ ), and interleukin-23 (IL-23). Otherwise, we added $\mathrm{CD} 4{ }^{+} \mathrm{T}$ cells to the culture plates that had been coated with $2 \mu \mathrm{g} / \mathrm{mL}$ anti-CD3 for Treg cell polarization. We, then, added soluble anti-CD28 $(1 \mu \mathrm{g} / \mathrm{mL})$ and cytokines, TGF- $\beta$ ( $2.5 \mathrm{ng} / \mathrm{mL})$, and IL-2 (20 ng/mL) to the culture plates and incubated them for 4 days to induce Treg cell differentiation. The differentiated Treg cells were then co-cultured with Ad-MSCs at a ratio of 1:4 from the 3rd to 5th passage for 4 days and we collected the supernatants for enzymelinked immunosorbent assays (ELISAs) and the cells for flow cytometry experiments and qPCR.

\section{Quantitative Real Time-Polymerase Chain Reaction (qPCR)}

We extracted total RNA from cultured cells using TRIzol. After measuring RNA concentrations and confirming their purities, we used $1 \mu \mathrm{g}$ RNA for reverse transcription to produce cDNA. We added cDNA samples to SYBR Green mixes for real-time PCR reactions (Table 1). qPCR reactions had final volumes of $20 \mu \mathrm{L}$ containing $1 \mu \mathrm{L}$ of the cDNA product, $1 \mu \mathrm{L}$ upstream primer, $1 \mu \mathrm{L}$ downstream primer, $\mathrm{ddH}_{2} \mathrm{O} 7 \mu \mathrm{L}$, and $10 \mu \mathrm{L} 2 \mathrm{x}$ Master mix. We used the Applied Biosystems 7500 Real Time-PCR system software to analyze qRT-PCR amplification products. We compared the expression levels of ROR $\gamma \mathrm{t}$, IL6R, IL-23R, LIF, LIFR between groups after normalizing their levels with the level of the gapdh housekeeping gene. Though we aimed to collect results from all participants, some samples did not have enough volume to run qPCR for all target genes. Therefore, the number of participants for some qPCRs were less than the total sample size.

\section{Measurement of Cytokines and LIF by ELISA}

We collected supernatants of cultured cells and measured levels of LIF, IL-6, IL-23, IL-17A, IL-10, and
Table I Sequences of the Primers for qRT-PCR

\begin{tabular}{|l|l|}
\hline Gene & Primer Sequences (5''-3') \\
\hline ROR & $\begin{array}{l}\text { Forward: TGTCCCGAGATGCTGTCAAGT } \\
\text { Reverse: GAGGGGTCTTGACCACTGGTT }\end{array}$ \\
\hline GAPDH & $\begin{array}{l}\text { Forward: GCCAGTAGACTCCACGACAT } \\
\text { Reverse: GCAAGTTCAACGGCACAG }\end{array}$ \\
\hline LIFR & $\begin{array}{l}\text { Forward: CCCCAAATAATGTTGAGGTTCTG } \\
\text { Reverse: CTGCGGCTGGGTTTGGTAT }\end{array}$ \\
\hline IL-6R & $\begin{array}{l}\text { Forward: CCTGCCAACATCACAGTCACTG } \\
\text { Reverse: CAGCCCGATATCTGAGCTCAA }\end{array}$ \\
\hline IL-23R & $\begin{array}{l}\text { Forward: CAAGAAACAGGCAAAAGGTA } \\
\text { Reverse: ATGTGACCTGGGGAACTGT }\end{array}$ \\
\hline LIF & $\begin{array}{l}\text { Forward: CAGCATCACTGAATCACAGAGC } \\
\text { Reverse: AGTATGAAACATCCCCACAGGG }\end{array}$ \\
\hline
\end{tabular}

TGF- $\beta$ in triplicate by following the protocol provided by the ELISA (eBioscience, San Diego, CA). The optical density (OD) values were measured at a 450 $\mathrm{nm}$ wavelength, and absorbance readings were then converted to concentrations of target proteins. We constructed a standard curve to calculate the concentrations of samples.

\section{Statistical Analysis}

We performed statistical analyses using SPSS 25.0 (IBM, Armonk, NY, USA) and GraphPad Prism 8.0 (GraphPad Software, San Diego, CA, USA) software. We used the $t$-test for parametric data. We expressed data as means \pm standard errors of means. We considered $p<0.05$ as statistically significant.

\section{Results}

\section{Demographic Information of PD Patients}

We obtained blood samples from 6 patients with PD to collect PBMCs for co-culture experiments. Table 2 lists the clinical features of these patients.

\section{Isolation of Ad-MSCs}

We confirmed the identity of the cultured Ad-MSCs by their flow cytometry expression profiles: they expressed CD44, CD29, CD73, CD105, CD13, and partially of CD49, but not CD31, CD34, or HLA-DR, as published. ${ }^{31,32}$ These results suggested that the cultured cells retained the characteristics of stem cells. 
Table 2 Clinical Features of Patients with PD

\begin{tabular}{|l|l|}
\hline \multicolumn{2}{|l|}{ Patients with Parkinson's Disease } \\
\hline Number & 6 \\
Gender (male/female) & $4 / 2$ \\
Age (years) & $44-75$ (mean 64.83) \\
Disease duration (years) & I-8 (mean 4.28) \\
& NA \\
UPDRS score & $8-20$ (mean 12.33) \\
\hline
\end{tabular}

\section{Ad-MSCs Inhibited the Differentiation of CD4 ${ }^{+} \mathrm{T}$ Cells into ThI 7 Cells}

We analyzed the influence of Ad-MSCs on the differentiation of CD4 ${ }^{+} T$ cells of patients with PD in vitro. When IL-1 $\beta$, IL-6, TGF- $\beta$ (10 ng/mL), IL-23 (40 ng/mL), anti-CD3, and anti$\mathrm{CD} 28$ monoclonal antibodies (mAbs) were added, $\mathrm{CD}^{+} \mathrm{T}$ cells were induced to differentiate into IL-17producing Th17 cells. After a 4-day co-culture with AdMSCs, the proportion of $\mathrm{IL}-17^{+} \mathrm{CD} 4^{+} \mathrm{Th} 17$ cells decreased significantly compared with that of the control cells that were grown without Ad-MSCs (Figure 1A and B). Also, the expression of ROR $\gamma \mathrm{t}$ (a Th17 specific transcription factor) was decreased significantly in the presence of Ad-MSCs (Figure 1C).

\section{Ad-MSCs Suppressed the Expression of IL-6R and IL-23R}

We observed decreased expressions of IL-6R and IL-23R mRNAs when $\mathrm{CD}^{+} \mathrm{T}$ cells were co-cultured with AdMSCs under the Th17 polarizing condition (Figure 2A and B), and decreased expression of IL-6R mRNA under the Treg polarizing condition (Figure $2 \mathrm{C}$ ). However, we found similar expressions of IL-6, IL-23, and TGF- $\beta$ when comparing cultures with non-MSCs treated $\mathrm{T}$ cells (Figure 2D-F). These results suggest that Ad-MSCs may inhibit the differentiation of Th17 cells by downregulating the expressions of ROR $\gamma \mathrm{t}$, IL-6R, and IL-23R mRNAs.

\section{Ad-MSCs Induced $C D 4^{+} T$ Cells into a Functional Treg Phenotype}

To analyze the influence of Ad-MSCs on $\mathrm{CD}^{+} \mathrm{T}$ cells, we measured the production of IL- 10 by $\mathrm{CD} 4^{+}$cells co-cultured under the Treg polarizing condition in the presence or absence of Ad-MSCs. We found the proportion of $\mathrm{CD} 4^{+} \mathrm{CD} 25^{+}$Foxp $^{+}{ }^{+} \mathrm{T}$ cells to be slightly increased after anti$\mathrm{CD} 3 / \mathrm{CD} 28$ Abs stimulation, but the proportion increased significantly under the Treg polarizing condition with the addition of Ad-MSCs (Figure 3A and B). Moreover, addition of Ad-MSCs markedly suppressed the expression of IL-6R mRNA (Figure 2C). After adding Ad-MSCs to the culture on day 4 , we observed a significant increase in the production of IL-10 in comparison with the production in the non-MSC treated cultures (Figure 3C). Therefore, Ad-MSCs showed capabilities of inducing $\mathrm{CD} 4^{+} \mathrm{T}$ cells into functional Treg cells and suppressing the differentiation into Th17 cells on day 4 under polarizing conditions.

\section{LIF is the Key Factor of the Inhibitory Effect}

LIF, an important cytokine secreted by stem cells, plays an anti-inflammatory role. ${ }^{33,34}$ We analyzed the levels of LIF to determine whether it (secreted by Ad-MSCs) was associated with the acquired immunosuppressive activities in our cultures. We found a significant increase in the level of LIF in the presence of Ad-MSCs compared to that when T cells were cultured alone under either the Th17 or the Treg polarizing condition (Figure 4A and B). We also observed increased expression of LIF mRNA, consistent with the increased production of LIF produced by the Ad-MSCs (Figure 4C and D). The expression of LIFR mRNA was
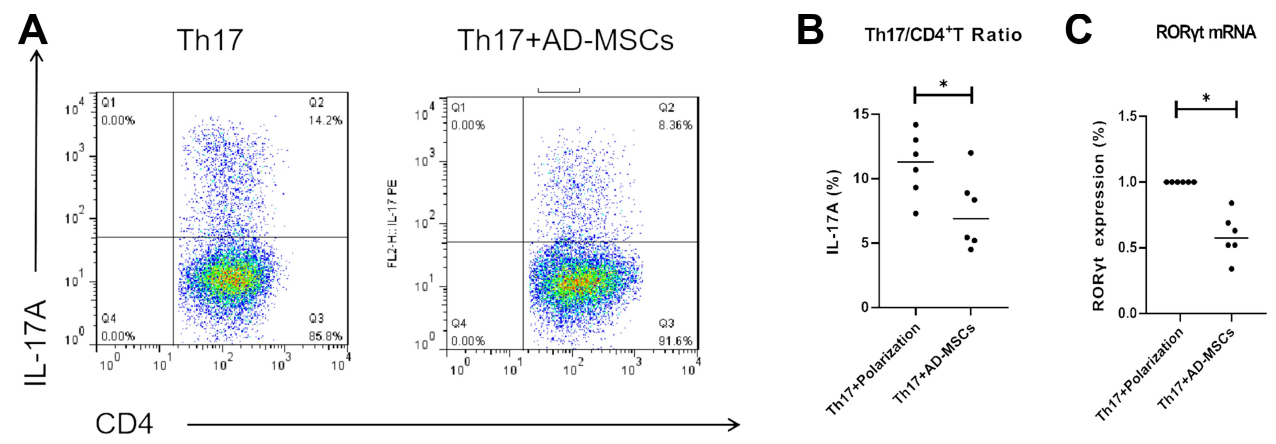

Figure I Ad-MSCs inhibited the differentiation of CD4 ${ }^{+}$T cells into ThI7 cells. Percentage of ThI7 (IL-I7A ${ }^{+} \mathrm{CD} 4^{+}$) cells analyzed by flow cytometry (A, B). After 4-day- coculture with Ad-MSCs, the level of ROR $\gamma \mathrm{t}$ mRNA was analyzed using real-time PCR (C). ${ }^{*} p<0.05$. 
IL-6R mRNA

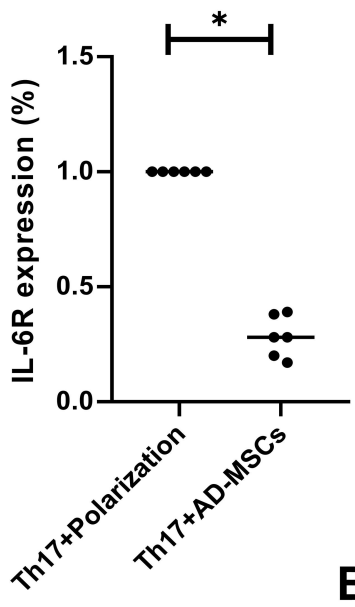

IL -6

D

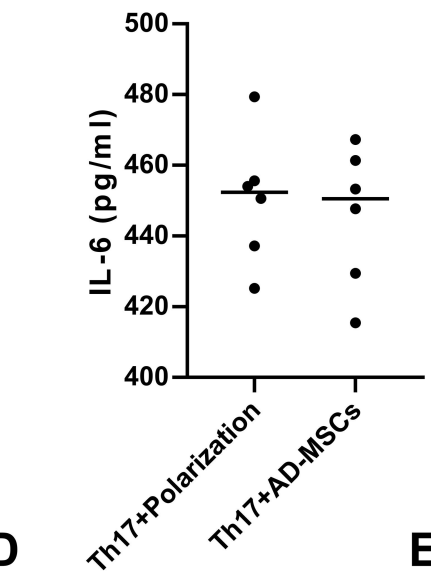

IL-23R mRNA

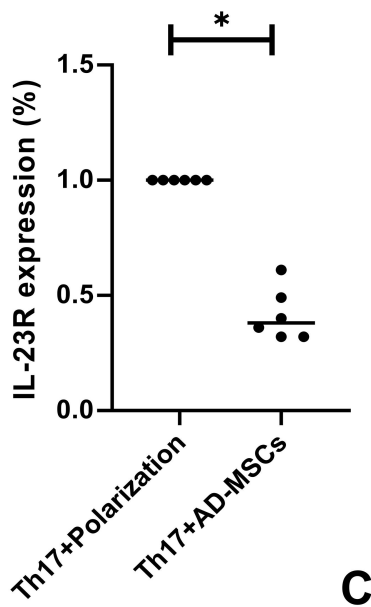

IL-23

E

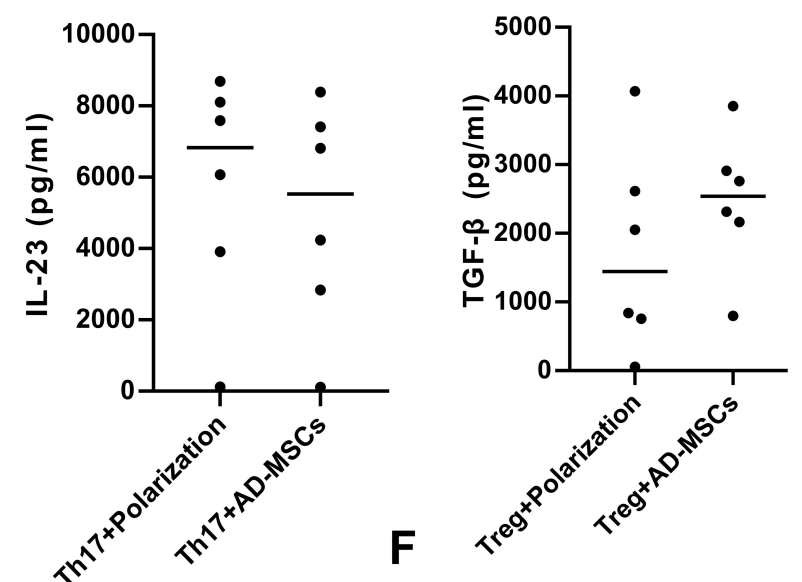

Figure 2 Ad-MSCs suppressed the expression of cytokines and their receptors. Low levels of expression of IL-6R and IL-23R mRNAs in co-cultures under the ThI7 polarized condition (A, B), and low levels of IL-6R mRNA under the Treg polarized condition (C, $n=3)$. Levels of IL-6, IL-23, and TGF- $\beta$ proteins showed similar results in $\mathrm{CD} 4^{+} \mathrm{T}$ cell co-cultures under either of the polarized conditions (D-F). ${ }^{*} p<0.05$.
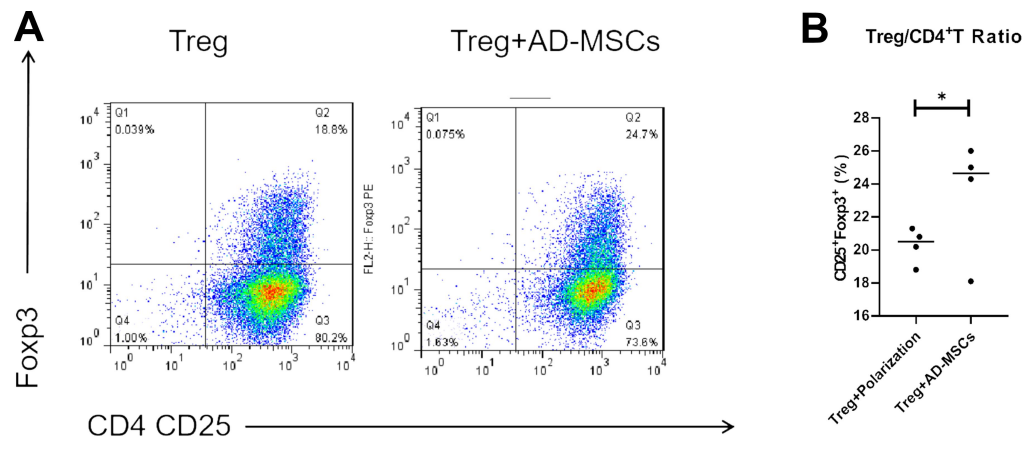

C IL-10

Figure 3 hAd-MSCs induced CD4+T cell differentiation into functional Treg cells. The proportion of Treg $\left(\mathrm{CD} 4^{+} \mathrm{CD} 25^{+}\right.$Foxp $\left.3^{+} \mathrm{T}\right)$ cells was analyzed by flow cytometry $(\mathbf{A}$; B, $n=4)$. Increased levels of IL- 10 in the supernatants of co-cultured systems as tested by ELISA (C). ${ }^{*} p<0.05$. 
LIF Th17

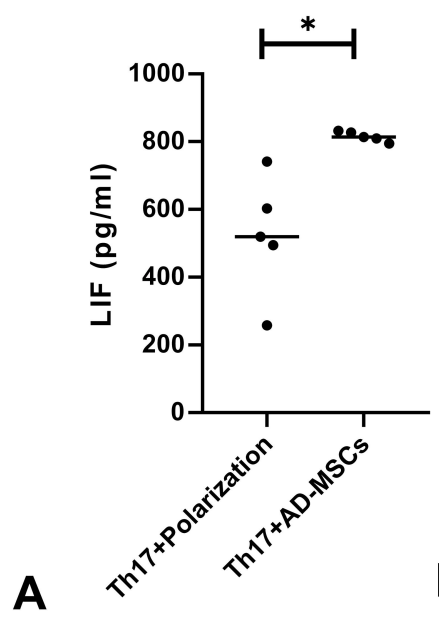

LIF mRNA Treg

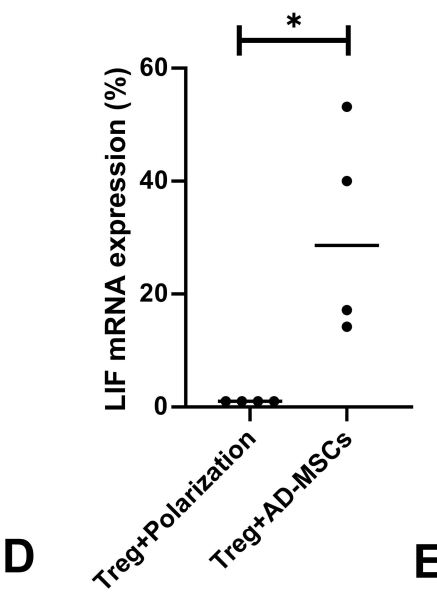

LIF Treg

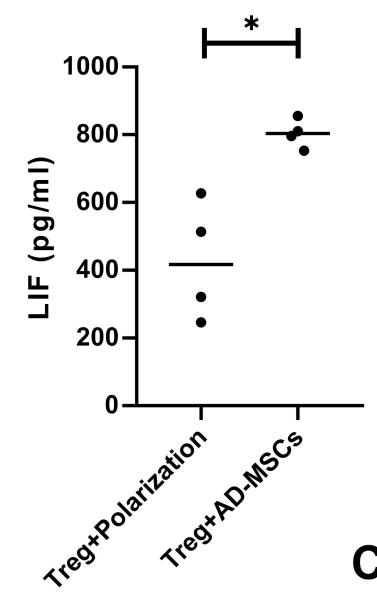

LIF mRNA Th17

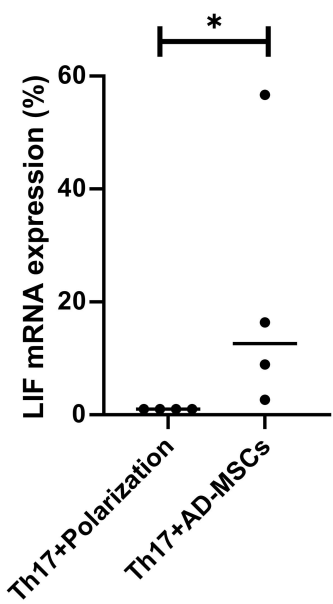

UFR mRNA Th17

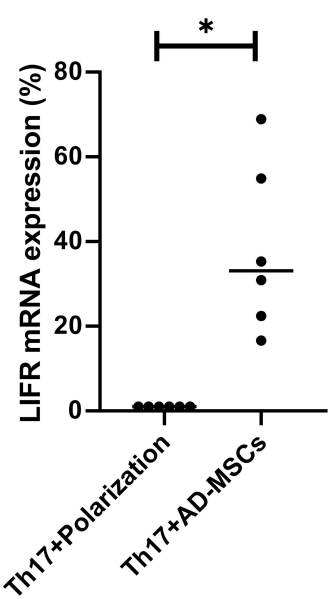

LIFR mRNA Treg

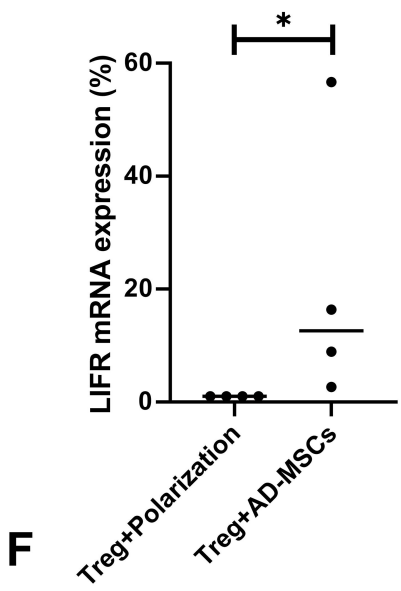

Figure 4 LIF is the key inhibitory factor. The levels of LIF protein were significantly upregulated in the supernatant of the co-cultured systems under either of the polarizing conditions tested by ELISA ( $\mathbf{A}, n=5 ; \mathbf{B}, n=4)$, real-time PCR results showed increased expression of LIF mRNA $(\mathbf{C}, n=4 ; \mathbf{D}, n=4)$. We found increased expressions of LIF-R mRNA by real-time PCR in co-cultures under both polarizing conditions $(\mathbf{E} ; \mathbf{F}, \mathrm{n}=4)$. ${ }^{*} p<0.05$.

increased under both Th17 and Treg polarizing conditions (Figure 4E and F). These results suggest that LIF may play a role in Ad-MSCs suppressing Th17 cell differentiation, promoting Treg differentiation, increasing the expression of LIFR mRNA, and suppressing the expression of IL-6R mRNA (Figure 2A and C).

\section{Discussion}

Human Ad-MSCs possess extraordinary properties attenuating effector $\mathrm{T}$ cell responses in diverse immune disorders. ${ }^{27-29}$ In this study, we found that human AdMSCs specifically inhibited IL-17-producing $\mathrm{CD} 4^{+} \mathrm{T}$ cell differentiation under the Th17 polarizing condition, and suppressed the expression of the transcription factor ROR $\gamma$ t. Ad-MSCs induced a functional $\mathrm{CD} 4^{+} \mathrm{CD} 25^{+} \mathrm{Foxp}^{+}{ }^{+}$Treg cell phenotype evidenced by the secretion of IL-10 under the Treg polarizing condition. These findings are in accordance with the expectation of a large number of studies searching for innovative therapeutics for patients with $\mathrm{PD} .^{12,18,30,35-37}$ 
Treg cell abnormalities in patients with PD have been shown either by reduced numbers or by functional deficits. ${ }^{4,5,17,18}$ These abnormalities may be due to increased numbers of Th17 cells, as well as increased secretion of IL-17 in the serum of patients with PD (Cen et al, 2017; Park et al, 2017). The blockage of IL-17 or neutralization with an IL-17 antibody promotes neuronal survival. ${ }^{5,6,9-11}$ Animal studies have revealed that an increased number of Th17 cells leads to tissue damage due to the production of the neurotoxic cytokine-interleukin IL-17, ${ }^{11-14,38}$ which is attenuated by Treg cells. ${ }^{12}$ Results of animal studies and clinical trials have suggested that Ad-MSCs are a potential PD therapeutic strategy modulating immune responses in patients with PD and animal models due to their suppression of Th17 responses and their induction of functional Treg cells..$^{24-29,39-41}$ We found that Ad-MSCs inhibited the differentiation of peripheral $\mathrm{CD} 4^{+} \mathrm{T}$ cells into Th17 cells and induced their differentiation into functional Treg cells, as evidenced by their resulting secretion of IL-10. Our results indicate that Ad-MSCs have the potential in regulating immune responses in vitro towards the balance between Th17 and Treg, which might be beneficial for PD patients.

The above phenomenon has been observed, ${ }^{29,39}$ but its underlying mechanisms remained unclear until now. Based on our previous findings showing that Ad-MSCs mediate immunosuppression of Th17 in patients with MS and a mouse model, ${ }^{31(\mathrm{p} 20), 32}$ we hypothesized that LIF is a key player mediating the suppression of Th17 cell differentiation. In our co-cultured Ad-MSCs-CD $4^{+} \mathrm{T}$ cell experiments, we found that Ad-MSCs accelerated the differentiation of $\mathrm{CD} 4^{+} \mathrm{T}$ cells towards Treg cells, as evidenced by the increased secretion of IL-10 under these conditions, and that they suppressed the differentiation towards IL-17-secreting Th17 cells, an assertion supported by the decreased expression of ROR $\gamma \mathrm{t}$. We also found that the LIF protein was significantly higher when $\mathrm{CD}^{+} \mathrm{T}$ cells were co-cultured with Ad-MSCs than when cultured alone under either the Th17 or Treg polarizing condition. Also, the expression of LIFR mRNA was increased under both Th17 and Treg polarizing conditions.

To further explore the mechanism for the suppression of T cell differentiation into Th17 by Ad-MSCs, we tested the levels of IL-23R and IL-6R. Both IL-23R and IL-6R are involved in the signaling pathways that lead to Th17 cell differentiation. ${ }^{21-23}$ Consistent with our hypothesis, the levels of IL-6R mRNA were downregulated under both Th17 and Treg polarizing conditions, and those of IL-23R mRNA were decreased under the Th17 polarizing condition. This was supported by the increased expression of ROR $\gamma \mathrm{t}$ and IL-6R mRNA after neutralizing LIF with an antibody as shown in our previous study. ${ }^{31}$ In this study, PBMCs from healthy controls were also tested when cocultured with Ad-MSCs. Ad-MSCs did not decrease the proportion of Th17 cells in control PBMCs to the same degree as observed in PBMCs of PD patients. LIF was also found to play a similar role as it did in the present study. These findings suggest that LIF is a key mediator of this process, and TGF- $\beta$ and PGE2 are involved in Treg upregulation and Th17 downregulation in humans. ${ }^{42}$

LIF belongs to the IL-6-type cytokine family and it is important for T-cell maturation. It is different from IL-6 because it binds to gp130/gp190 heterodimers, whereas IL-6 binds to gp130 homodimers. ${ }^{43} \mathrm{Gp} 130$ is the shared subunit of the cytokine receptors of the IL-6 family. IL-6 inhibits the expression of the LIFR subunit, opposing the physiological effect of LIF. ${ }^{43,44}$ This may explain the increased LIFR levels observed in our study.

LIF suppresses the expression of Th17 specific genes such as the transcription factor ROR $\gamma \mathrm{t}$, and it simultaneously increases the expression of the Treg cell-specific transcription factor-Foxp3. ${ }^{33,34,44}$ In contrast, IL-6 decreases the level of Foxp3 and increases the level of ROR $\gamma$ t. $^{21,44}$ Therefore, a LIF/IL-6 balance is critical for T-cell lineage maturation. ${ }^{43}$ These results suggest that LIF, in combination with IL-6 signal pathway blockers, may be part of therapeutic approaches for PD and other immune disorders, ${ }^{34}$ even if the underlying mechanism of PD pathogenesis remains largely unknown.

The present study has its strength and limitations. We found that Ad-MSCs derived from humans promotes differentiation of $\mathrm{CD}^{+} \mathrm{T}$ cells into Treg by increasing the level of LIF and decreasing the level of IL6, IL6R., and ROR $\gamma$ t, suggesting that Ad-MSCs and LIF are potential therapeutics for pathological conditions involving an imbalance of Th17 and Treg. However, an important limitation of our study is a lack of control group. A four group study comparing PD PBMCs + PD Ad-MSCs, PD PBMCs + healthy Ad-MSCs, healthy PBMCs + PD Ad-MSCs, and healthy PBMCs + healthy Ad-MSCs would have provided a comprehensive understanding of the interactions between PBMCs and Ad-MSCs. Also, the present study did not test separately whether LIF is sufficient to induce Treg differentiation without adding Ad-MSCs in vitro. A future study shall be planned to address this concerns. Lastly, due to limitations of sampling, qPCR for all target genes was not carried out for the entire sample. 


\section{Conclusion}

The present study demonstrated that human Ad-MSCs suppress the differentiation of $\mathrm{CD} 4^{+} \mathrm{T}$ cells (isolated from patients with PD) into Th17 cells in vitro. This suppressive effect was mainly associated with an increase in functional $\mathrm{CD} 4^{+} \mathrm{CD} 25^{+}$Foxp $^{+}{ }^{+}$Treg cells and IL-10 secretion. Within the limitations of our study, our results suggest that AdMSCs may be a candidate for regulating inflammatory responses and promoting neuroprotection for PD. However, this needs to be confirmed by further studies utilizing a control group.

\section{Ethical Clearance}

The Human Research Ethics Committee of our hospital approved the procedures for this study (WYYY2014-001) and we obtained written informed consent from all the donors. This study was conducted in accordance with the Declaration of Helsinki.

\section{Acknowledgments}

This manuscript has been released as a pre-print at research square, Yong Bi et al. ${ }^{45}$

\section{Author Contributions}

All authors made a significant contribution to the work reported, whether that is in the conception, study design, execution, acquisition of data, analysis and interpretation, or in all these areas; took part in drafting, revising or critically reviewing the article; gave final approval of the version to be published; have agreed on the journal to which the article has been submitted; and agree to be accountable for all aspects of the work.

\section{Funding}

This study was supported by a grant from the Commission of Health and Family Planning, Hongkou District, Shanghai, awarded to Dr. Yong Bi (No. 1701-02), a grant from Zhejiang Natural Science Foundation (LY15H090019), a grant from Wenzhou Scientific Planning (Y20140684), and a grant from the State Key Open Projects of Soochow University (KJS1726).

\section{Disclosure}

$\mathrm{Dr} \mathrm{Xu}$ Zhang reports non-financial support from Wenzhou Medical University First Affiliated Hospital, during the conduct of the study; non-financial support from Wenzhou medical University First Affiliated Hospital, outside the submitted work. The authors declare no other conflicts of interest in this work.

\section{References}

1. Hughes AJ, Daniel SE, Kilford L, Lees AJ. Accuracy of clinical diagnosis of idiopathic Parkinson's disease: a clinico-pathological study of 100 cases. J Neurol Neurosurg Psychiatry. 1992;55 (3):181-184. doi:10.1136/jnnp.55.3.181

2. Kalia LV, Lang AE. Parkinson's disease. Lancet. 2015;386 (9996):896-912. doi:10.1016/S0140-6736(14)61393-3

3. Brochard V, Combadière B, Prigent A, et al. Infiltration of CD4+ lymphocytes into the brain contributes to neurodegeneration in a mouse model of Parkinson disease. J Clin Invest. 2009;119 (1):182-192. doi:10.1172/JCI36470

4. Saunders JAH, Estes KA, Kosloski LM, et al. CD4+ regulatory and effector/memory t cell subsets profile motor dysfunction in Parkinson's disease. J Neuroimmune Pharmacol. 2012;7(4):927-938. doi:10.1007/ s11481-012-9402-z

5. Cen L, Yang C, Huang S, et al. Peripheral lymphocyte subsets as a marker of Parkinson's disease in a Chinese population. Neurosci Bull. 2017;33(5):493-500. doi:10.1007/s12264-017-0163-9

6. Park J, Lee J-W, Cooper SC, Broxmeyer HE, Cannon JR, Kim CH. Parkinson disease-associated LRRK2 G2019S transgene disrupts marrow myelopoiesis and peripheral Th17 response. J Leukoc Biol. 2017;102(4):1093-1102. doi:10.1189/jlb.1A0417-147RR

7. Prinz M, Priller J. The role of peripheral immune cells in the CNS in steady state and disease. Nat Neurosci. 2017;20(2):136-144. doi:10. 1038/nn.4475

8. Sulzer D, Alcalay RN, Garretti F, et al. T cells from patients with Parkinson's disease recognize $\alpha$-synuclein peptides. Nature. 2017;546 (7660):656-661. doi:10.1038/nature22815

9. Storelli E, Cassina N, Rasini E, Marino F, Cosentino M. Do Th17 lymphocytes and IL-17 contribute to Parkinson's disease? A systematic review of available evidence. Front Neurol. 2019;10.

10. Sommer A, Marxreiter F, Krach F, et al. Th17 lymphocytes induce neuronal cell death in a human iPSC-based model of Parkinson's disease. Cell Stem Cell. 2018;23(1):123-131.e6. doi:10.1016/j. stem.2018.06.015

11. Liu Z, Qiu A-W, Huang Y, et al. IL-17A exacerbates neuroinflammation and neurodegeneration by activating microglia in rodent models of Parkinson's disease. Brain Behav Immun. 2019;81:630-645. doi:10.1016/j.bbi.2019.07.026

12. Reynolds AD, Stone DK, Hutter JAL, Benner EJ, Mosley RL, Gendelman HE. Regulatory T cells attenuate Th17 cell-mediated nigrostriatal dopaminergic neurodegeneration in a model of Parkinson's disease. J Immunol. 2010;184(5):2261-2271. doi:10.4049/jimmunol.0901852

13. Liu Z, Huang Y, Cao -B-B, Qiu Y-H, Peng Y-P. Th17 cells induce dopaminergic neuronal death via LFA-1/ICAM-1 interaction in a mouse model of Parkinson's disease. Mol Neurobiol. 2017;54 (10):7762-7776. doi:10.1007/s12035-016-0249-9

14. Dutta D, Kundu M, Mondal S, et al. RANTES-induced invasion of Th17 cells into substantia nigra potentiates dopaminergic cell loss in MPTP mouse model of Parkinson's disease. Neurobiol Dis. 2019;132:104575. doi:10.1016/j.nbd.2019.104575

15. Reynolds AD, Stone DK, Mosley RL, Gendelman HE. Proteomic studies of nitrated alpha-synuclein microglia regulation by CD4+CD25+ T cells. J Proteome Res. 2009;8(7):3497-3511. doi:10.1021/pr9001614

16. Huang Y, Liu Z, Cao -B-B, Qiu Y-H, Peng Y-P. Treg cells protect dopaminergic neurons against MPP+ neurotoxicity via CD47-SIRPA interaction. Cell Physiol Biochem. 2017;41(3):1240-1254. doi:10. $1159 / 000464388$

17. Chen Y, Qi B, Xu W, et al. Clinical correlation of peripheral CD4 +-cell sub-sets, their imbalance and Parkinson's disease. Mol Med Rep. 2015;12(4):6105-6111. doi:10.3892/mmr.2015.4136 
18. Gendelman HE, Zhang Y, Santamaria P, et al. Evaluation of the safety and immunomodulatory effects of sargramostim in a randomized, double-blind Phase 1 clinical Parkinson's disease trial. NPJ Parkinsons Dis. 2017;3(1):10. doi:10.1038/s41531-017-0013-5

19. Duffy SS, Keating BA, Perera CJ, Moalem-Taylor G. The role of regulatory $\mathrm{T}$ cells in nervous system pathologies. $J$ Neurosci Res. 2018;96(6):951-968. doi:10.1002/jnr.24073

20. Álvarez-Luquín DD, Arce-Sillas A, Leyva-Hernández J, et al. Regulatory impairment in untreated Parkinson's disease is not restricted to Tregs: other regulatory populations are also involved. J Neuroinflammation. 2019;16(1):212. doi:10.1186/s12974-019-1606-1

21. Zhu J, Yamane H, Paul WE. Differentiation of effector CD4 T cell populations. Annu Rev Immunol. 2010;28(1):445-489. doi:10.1146/ annurev-immunol-030409-101212

22. Littman DR, Rudensky AY. Th17 and regulatory T cells in mediating and restraining inflammation. Cell. 2010;140(6):845-858. doi:10.10 16/j.cell.2010.02.021

23. Weaver CT, Hatton RD. Interplay between the T H 17 and T Reg cell lineages: a (co-)evolutionary perspective. Nat Rev Immunol. 2009;9 (12):883-889. doi:10.1038/nri2660

24. Chang K-A, Lee J-H, Suh Y-H. Therapeutic potential of human adipose-derived stem cells in neurological disorders. J Pharmacol Sci. 2014;126(4):293-301. doi:10.1254/jphs.14R10CP

25. Shi Y, Wang Y, Li Q, et al. Immunoregulatory mechanisms of mesenchymal stem and stromal cells in inflammatory diseases. Nat Rev Nephrol. 2018;14(8):493-507. doi:10.1038/s41581-018-0023-5

26. Darlington PJ, Boivin M-N, Renoux C, et al. Reciprocal Th1 and Th17 regulation by mesenchymal stem cells: implication for multiple sclerosis. Ann Neurol. 2010;68(4):540-545. doi:10.1002/ana.22065

27. Panés J, García-Olmo D, Van Assche G, et al. Expanded allogeneic adipose-derived mesenchymal stem cells (Cx601) for complex perianal fistulas in Crohn's disease: a Phase 3 randomised, double-blind controlled trial. Lancet. 2016;388(10051):1281-1290. doi:10.1016/ S0140-6736(16)31203-X

28. Álvaro-Gracia JM, Jover JA, García-Vicuña R, et al. Intravenous administration of expanded allogeneic adipose-derived mesenchymal stem cells in refractory rheumatoid arthritis (Cx611): results of a multicentre, dose escalation, randomised, single-blind, placebo-controlled phase Ib/IIa clinical trial. Ann Rheum Dis. 2017;76(1):196-202. doi:10.1136/annrheumdis-2015-208918

29. Wang D, Huang S, Yuan X, et al. The regulation of the Treg/Th17 balance by mesenchymal stem cells in human systemic lupus erythematosus. Cell Mol Immunol. 2017;14(5):423-431. doi:10. 1038/cmi.2015.89

30. Prots I, Winner B. Th17 cells: a promising therapeutic target for Parkinson's disease? Expert Opin Ther Targets. 2019;23 (4):309-314. doi:10.1080/14728222.2019.1590336

31. Xiao-bin LIN. Adipose tissue-derived stem cells mediated immunosuppression on Th17 in multiple sclerosis. Chin J Pathophysiol. 2016;32(1):51-57.

32. Jia L, Chen Y, Chen Z, et al. Therapeutic effects of human adipose tissue-derived stem cell (hADSC) transplantation on experimental autoimmune encephalomyelitis (EAE) mice. Sci Rep. 2017;7 (1):42695. doi:10.1038/srep42695

Clinical Interventions in Aging

\section{Publish your work in this journal}

Clinical Interventions in Aging is an international, peer-reviewed journal focusing on evidence-based reports on the value or lack thereof of treatments intended to prevent or delay the onset of maladaptive correlates of aging in human beings. This journal is indexed on PubMed Central, MedLine, CAS, Scopus and the Elsevier
33. Cao W, Yang Y, Wang Z, et al. Leukemia inhibitory factor inhibits $\mathrm{T}$ helper 17 cell differentiation and confers treatment effects of neural progenitor cell therapy in autoimmune disease. Immunity. 2011;35 (2):273-284. doi:10.1016/j.immuni.2011.06.011

34. Janssens K, Van den Haute C, Baekelandt V, et al. Leukemia inhibitory factor tips the immune balance towards regulatory $\mathrm{T}$ cells in multiple sclerosis. Brain Behav Immun. 2015;45:180-188. doi:10.1016/j.bbi.2014.11.010

35. Athauda D, Foltynie T. The ongoing pursuit of neuroprotective therapies in Parkinson disease. Nat Rev Neurol. 2015;11(1):25-40. doi:10.1038/nrneurol.2014.226

36. Kalia LV, Kalia SK, Lang AE. Disease-modifying strategies for Parkinson's disease. Mov Disord. 2015;30(11):1442-1450. doi:10. $1002 /$ mds. 26354

37. Schutt CR, Gendelman HE, Mosley RL. Tolerogenic bone marrowderived dendritic cells induce neuroprotective regulatory $\mathrm{T}$ cells in a model of Parkinson's disease. Mol Neurodegener. 2018;13(1):26. doi:10.1186/s13024-018-0255-7

38. Peters A, Pitcher LA, Sullivan JM, et al. Th17 cells induce ectopic lymphoid follicles in central nervous system tissue inflammation. Immunity. 2011;35(6):986-996. doi:10.1016/j.immuni.2011.10.015

39. Ghannam S, Pène J, Moquet-Torcy G, Torcy-Moquet G, Jorgensen C, Yssel H. Mesenchymal stem cells inhibit human Th17 cell differentiation and function and induce a $\mathrm{T}$ regulatory cell phenotype. J Immunol. 2010;185(1):302-312. doi:10.4049/jimmunol.0902007

40. Kwon M-S, Noh M-Y, Oh K-W, et al. The immunomodulatory effects of human mesenchymal stem cells on peripheral blood mononuclear cells in ALS patients. $J$ Neurochem. 2014;131(2):206-218. doi:10. 1111/jnc. 12814

41. Baharlou R, Ahmadi-Vasmehjani A, Faraji F, et al. Human adipose tissue-derived mesenchymal stem cells in rheumatoid arthritis: regulatory effects on peripheral blood mononuclear cells activation. Int Immunopharmacol. 2017;47:59-69. doi:10.1016/j.intimp.2017.03. 016

42. Singer NG, Caplan AI. Mesenchymal stem cells: mechanisms of inflammation. Аnnu Rev Pathol. 2011;6(1):457-478. doi:10.1146/ annurev-pathol-011110-130230

43. Metcalfe SM. LIF in the regulation of T-cell fate and as a potential therapeutic. Genes Immun. 2011;12(3):157-168. doi:10.1038/ gene.2011.9

44. Gao W, Thompson L, Zhou Q, et al. Treg versus Th17 lymphocyte lineages are cross-regulated by LIF versus IL-6. Cell Cycle. 2009;8 (9):1444-1450. doi:10.4161/cc.8.9.8348

45. $\mathrm{Bi} \mathrm{Y}$, Lin $\mathrm{X}$, Liang $\mathrm{H}$, et al. Human Adipose Tissue-Derived Mesenchymal Stem Cells in Parkinson's disease: Inhibit T Helper 17 Cell Differentiation and Regulate Immune Balance Towards a Regulatory T Cell Phenotype. 2020.
Bibliographic databases. The manuscript management system is completely online and includes a very quick and fair peer-review system, which is all easy to use. Visit http://www.dovepress.com/ testimonials.php to read real quotes from published authors. 